Mens

revue d'histoire intellectuelle de l'Amérique française

\title{
Souvenir, mémoire et imaginaire de la francité dans Les Filles de Caleb
}

\section{Frédéric Demers}

Volume 7, numéro 1, automne 2006

URI : https://id.erudit.org/iderudit/1024223ar

DOI : https://doi.org/10.7202/1024223ar

Aller au sommaire du numéro

Éditeur(s)

Centre de recherche en civilisation canadienne-française

ISSN

1492-8647 (imprimé)

1927-9299 (numérique)

Découvrir la revue

Citer cet article

Demers, F. (2006). Souvenir, mémoire et imaginaire de la francité dans Les Filles de Caleb. Mens, 7(1), 73-115. https://doi.org/10.7202/1024223ar

\section{Résumé de l'article}

La télésérie Les Filles de Caleb, œuvre de fiction inspirée de la vie de personnes réelles, a proposé aux Québécois une représentation d'eux-mêmes qui misait sur une panoplie de stéréotypes bien établis dans leur imaginaire et à laquelle ils ont conféré une valeur d'authenticité à la fois historique et nationale. Par une analyse de son discours sur la francité, notion entendue comme patrimoine culturel hérité de France ou tenu pour tel dans la mémoire, cet article montre comment la télésérie s'est servie de ces stéréotypes pour donner à son récit une valeur ajoutée sur le plan identitaire. La réaction populaire suscitée par Les Filles de Caleb témoigne avec éloquence de la réussite de l'entreprise, tout autant qu'elle nous permet de pénétrer à l'intérieur du mécanisme de construction de la réalité à partir du contenu d'une fiction.
Ce document est protégé par la loi sur le droit d'auteur. L'utilisation des services d'Érudit (y compris la reproduction) est assujettie à sa politique d'utilisation que vous pouvez consulter en ligne.

https://apropos.erudit.org/fr/usagers/politique-dutilisation/ 


\title{
SOUVENIR, MÉMOIRE ET IMAGINAIRE DE LA FRANCITÉ DANS LES FILLES DE CALEB ${ }^{1}$
}

\author{
Frédéric Demers \\ Département d'histoire \\ Université Laurentienne
}

\section{Résumé}

La télésérie Les Filles de Caleb, œuvre de fiction inspirée de la vie de personnes réelles, a proposé aux Québécois une représentation d'euxmêmes qui misait sur une panoplie de stéréotypes bien établis dans leur imaginaire et à laquelle ils ont conféré une valeur d'authenticité à la fois historique et nationale. Par une analyse de son discours sur la francité, notion entendue comme patrimoine culturel hérité de France ou tenu pour tel dans la mémoire, cet article montre comment la télésérie s'est servie de ces stéréotypes pour donner à son récit une valeur ajoutée sur le plan identitaire. La réaction populaire suscitée par Les Filles de Caleb témoigne avec éloquence de la réussite de l'entreprise, tout autant qu'elle nous permet de pénétrer à l'intérieur du mécanisme de construction de la réalité à partir du contenu d'une fiction.

\section{Abstract}

The mini-series Les Filles de Caleb, a work of fiction inspired by actual persons, provided Quebecers with a self-representation moulded out of a variety of stereotypes that were entrenched in Quebec's national imagination and were viewed as historically authentic. By analysing the discourse of francite, a notion which refers to Quebec's French cultural legacy or to aspects of Quebec's cultural beritage that are assumed to have been derived from France, this article shows how the mini-series used stereotypes to lend authenticity to its narrative in the 
realm of identity. The success of Les Filles de Caleb also illustrates the extent to which reality can be constructed out of fiction.

La télésérie Les Filles de Caleb a connu un succès fulgurant au début des années 1990. Dans le but de comprendre un peu mieux les ressorts de l'assiduité passionnée que cette histoire profondément tragique avait suscitée au sein de la population du Québec, nous en avons analysé le contenu et la réception ${ }^{2}$. L'angle retenu fut celui de l'imaginaire national et historique. Distinct des souvenirs, qui sont des fragments de passé existant en esprit, et de la mémoire, cette faculté humaine qui active les souvenirs, les ordonne, leur injecte un sens ou les enfouit, l'imaginaire nous avait semblé trouver dans la métaphore du réseau une expression fort heureuse, respectueuse à la fois de sa complexité et du mouvement qui le caractérise. Comme réseau, comme système générateur de sens, l'imaginaire accueille des mémoires particulières multiples et changeantes selon les générations, les conditions socioéconomiques ou encore le bagage de référents culturels de leurs porteurs. Rendre compte d'un imaginaire n'est ainsi jamais simple ; un risque existe toujours de confondre l'imaginaire des créateurs et celui de la société tout entière. En fait, on se voit même parfois forcé de s'en tenir à l'imaginaire de ceux qui s'expriment, faute de pouvoir accéder à l'univers des autres groupes sociaux. Il faut alors essayer de déterminer si cet imaginaire avait fertilisé le reste de la société et, le cas échéant, jusqu'à quel point. L'imaginaire semble intéresser de plus en plus de chercheurs québécois depuis quelques années et ce fut notre intention, avec la thèse dont cet article découle, de participer activement à ce mouvement ${ }^{3}$.

L'élaboration de notre grille d'analyse nous a conduit à distinguer trois pôles culturels au cœur de l'imaginaire national et historique des Québécois d'héritage canadien-français : 
a) la francité, b) la canadianité, et c) la continentalitét. Chacun réunit, pêle-mêle, des valeurs, des faits de société, des attitudes, des mentalités. Pour dire les choses brièvement, ces trois pôles correspondent respectivement à a) un patrimoine culturel hérité de France, à préserver et à transmettre, b) un patrimoine trouvé au Nouveau Monde et approprié par et pour soi, et c) un patrimoine reçu avec plus ou moins de bonheur, imposé à soi par les circonstances ou les forces du changement ${ }^{5}$. Sous certains de ces aspects et à cause de sa dimension imposée, la continentalité contribue également à vivifier la conscience des Québécois d'héritage canadien-français de leur statut de minoritaires. Une tension perpétuelle existe entre ces trois pôles, au centre de laquelle s'inscrit le sujet politique franco-québécois. Chacun exerce une force gravitationnelle sur lui, mais tous trois s'équilibrent et contribuent à maintenir le sujet en place . $^{6}$

Selon nous, la télésérie Les Filles de Caleb a acquis aux yeux de son public un capital symbolique enviable principalement parce que son récit s'est déployé au cœur même de cet espace de l'imaginaire ordonné selon les trois pôles mentionnés plus haut ${ }^{7}$. Une impression de très forte authenticité s'est dégagée de cette œuvre qui, pour plusieurs, leva le voile sur quelque chose de vrai, quelque chose allant au-delà de la fiction ou la transcendant. L'authenticité perçue fut à la fois historique ("ça se passait vraiment comme ça dans l'ancien temps») et nationale ("c'est vraiment nous, notre âme, l'être collectif franco-québécois qu'on voit à l'écran »). Les Filles de Caleb offrit aux Québécois bien plus qu'une relation des amours tumultueuses entre Émilie Bordeleau (Marina Orsini), la fière institutrice, et Ovila Pronovost (Roy Dupuis), son ancien élève; elle leur fit don d'une représentation globale et cohérente de leur passé en jouant sur une multitude de tableaux en même temps. Vieux Canada français et Québec post- 
1960, nomadisme et sédentarité, catholicisme et laïcité, ruralité et urbanité, tous ces thèmes et bien d'autres en lien avec nos trois pôles culturels s'y sont entremêlés sans pour autant que leurs valeurs signifiées y soient toujours identifiées de manière positive ou négative. La chose mérite qu'on insiste : aucun parti pris d'ensemble ne favorise ce qui relève de l'ancien ou du neuf, ou encore de l'un des trois pôles. Du point de vue de l'imaginaire national et historique, ce fut plutôt une remarquable synthèse que la télésérie opéra. Si les souvenirs du passé que cette dernière a éveillés furent nombreux, la mémoire qu'elle en a suggérée demeura axiologiquement neutre, en ce sens qu'elle s'abstint de trancher entre divers champs sémiotiques.

Nous présenterons ici, à tour de rôle, la mémoire de la francité construite par Les Filles de Caleb ainsi que la réaction populaire et critique à la télésérie telle que nous avons pu la reconstituer. À partir de cet échantillon qu'est la francité, nous montrerons quel usage a fait la télésérie d'un éventail de thèmes clés, de certaines sensibilités fortes pour nouer sa trame narrative. Nous sommes conscient qu'en l'isolant comme nous nous apprêtons à le faire, nous lui retirons une partie de sa puissance évocatrice : les bienfaits du monde rural, par exemple, maintes fois réitérés en mots et en image, n'ont pris tous leur sens qu'en relation avec la misère urbaine vécue par les principaux protagonistes dans les deux derniers épisodes ${ }^{8}$. L'exercice n'en sera pas vain pour autant, croyons-nous, parce qu'il permettra quand même d'illustrer comment et pourquoi la télésérie a pu susciter un tel engouement.

\section{Comme un parfum d'authenticité}

À certains égards, la facture télévisuelle des Filles de Caleb fait penser à certaines toiles inspirées de l'Antiquité peintes en Europe à l'époque où y triomphait le classicisme, des 
toiles dans lesquelles des protagonistes drapés de vêtements anormalement propres aux coloris chatoyants animent des scènes ayant en fin de compte peu à voir avec le réel crasseux de la vie quotidienne au temps de la civilisation gréco-romaine. Avec plus de subtilité, la télésérie met en scène un monde révolu en usant de représentations quelque peu figées, mais intériorisées par le public franco-québécois. Le récit des Filles de Caleb exhale une odeur diffuse de francité. Langue, terroir, famille nombreuse, catholicisme... : tous les suspects usuels s'y retrouvent pêle-mêle. Mais il ne s'agit que de cela : un parfum. Nous entendons par là que, parmi tous les éléments constitutifs de la francité qui se découvrent au fil des épisodes de la télésérie, aucun ne possède de valeur dramatique en soi. Aucun ne se retrouve au centre d'un enjeu à quelque moment que ce soit. Aucun ne se révèle la cause directe ou exclusive d'un affrontement entre deux protagonistes. La francité aromatise artificiellement le récit ou, pour user d'une image plus collée à l'univers de la télévision, elle agit comme un décor pour la narration. Cela n'a pas pour autant l'effet de rendre ses composantes insignifiantes ou anodines. Au contraire, toutes servent le même dessein de renforcer l'impression d'authenticité de la représentation d'ensemble.

\section{La langue française}

Le thème de la langue française passe principalement par l'entremise du personnage de l'inspecteur scolaire Henri Douville (Jacques Lussier). Chez lui, la langue devient objet de passion, d'investissement émotionnel et d'affirmation identitaire. Henri porte en lui l'amour du français et manifeste un souci constant de le promouvoir et d'en préserver la richesse et la beauté, encore qu'il le fasse d'une manière un peu caricaturale? 
Le purisme linguistique de l'inspecteur se manifeste en toutes circonstances, même quand les circonstances ne l'invitent pas. Un jour de l'été 1898 , venu les bras chargés de cadeaux rendre visite à l'institutrice Émilie qu'il courtise ouvertement, il parle sa langue précieuse habituelle avec la famille Bordeleau, car c'est la seule qu'il connaisse. Alors qu'un habitant, offrant ses présents, se serait peut-être contenté de dire laconiquement : «C'est pour le dérangement », Henri appuie sur chaque mot de toute sa préciosité : "Je ne pouvais tout de même pas, chère Émilie, déranger votre famille dans son précieux repos dominical sans me faire excuser. Même en tenant compte de votre bienveillante tolérance! » (épisode 8) Plus tard, exprimant sa flamme par écrit, il confiera à Émilie dans son même style ampoulé son désir «de grimper sur les toits pour clamer au monde que nul automne ne saurait être aussi beau que vos cheveux ", etc., etc. (épisode 9) Au fil de la télésérie, chaque scène où apparaît Henri devient l'occasion d'un fervent hommage au génie de la langue française.

Le souci de l'inspecteur de parler un français idoine va de pair avec celui de transmettre aux autres une langue de qualité. S'étant permis, à l'occasion de sa visite annuelle marquant la fin du calendrier scolaire, d'employer le verbe bourlinguer dans la dictée qu'il donne aux élèves, Henri se hâte, en s'accompagnant d'un geste de la main à la fois paternel et sécurisant, de clarifier la situation : bourlinguer est certes " un mot d'argot », mais " toléré par l'Académie [française] », donc acceptable (épisode 8). Le soir venu, malgré sa volonté de faire naitre une atmosphère intimiste à l'occasion du repas qu'il partage avec Émilie, Henri ne parvient pas à se retenir de corriger l'institutrice quand celle-ci s'échappe et laisse tomber un malheureux « $j$ 'avais pas». 
«Excusez mon impertinence, chère demoiselle, mais comme vous avez pu le constater, je voue un culte presque païen à la langue française, explique-t-il pour se faire pardonner. » Voyant qu'il a froissé Émilie, il enchaîne : "Il ne faut pas m'en vouloir. J'ai passé ma vie en institution. D'abord chez les religieuses, ensuite chez les jésuites. Cela m'a rendu tatillon. Peut-être même un peu maniaque. » Mais il y a plus : "La langue, ajoute Henri, c'est mon seul héritage. »

Le français est à ce jour tout ce que l'inspecteur a reçu de son enfance difficile - il a grandi en orphelinat - , mais aussi tout ce qu'il, encore célibataire, peut espérer léguer. Chaque chose, chez lui, y ramène. Pas étonnant alors qu'il l'adore au point de lui rendre " un culte ", qui plus est "presque païen ", c'est-à-dire presque caractérisé par des excès. Valoriser la langue, c'est se valoriser soi-même. Le français n'est pas simplement inscrit au cœur de l'identité personnelle d'Henri ; il constitue l'essence même de son être. À ce titre, le souci constant que manifeste l'inspecteur de transmettre une langue de qualité à ses interlocuteurs n'est rien d'autre que la manifestation de son désir de se perpétuer.

\section{Le catholicisme et la dévotion}

On sait l'importance du religieux au sein de la société rurale canadienne-française du début du $\mathrm{XX}^{\mathrm{e}}$ siècle. Sans surprise compte tenu du souci de la télésérie d'offrir une reconstitution vraisemblable du passé, les manifestations du catholicisme reviennent régulièrement dans les épisodes. Quatre angles sont tour à tour mis en valeur : a) la place de la religion dans le quotidien, b) son rôle en tant que ciment social, c) sa fonction de soutien dans les épreuves, et $d$ ) l'image des représentants de l'Église.

Les premières minutes de l'épisode initial lèvent le voile sur la manière dont le catholicisme participe à la vie de tous 
les jours des personnages. Caleb Bordeleau (Germain Houde), le père d'Émilie, est celui par l'entremise duquel la narration inscrit ce thème à son programme. Tout d'abord, en colère contre sa fille Émilie qui conteste son autorité sous le toit familial, Caleb l'envoie dans sa chambre en pénitence et, assénant une sentence digne d'un clerc, lui ordonne de dire son " acte de contrition en pensant au Quatrième Commandement : père et mère tu honoreras " (épisode 1). Le religieux se manifeste de nouveau quelques instants plus tard. Discutant avec sa femme Célina (Johanne-Marie Tremblay), Caleb, qui s'est vu forcé d'abattre son étalon plus tôt dans la soirée, compare sa déconfiture à celle de "Samson, le jour où il s'est fait couper les cheveux ». Le lendemain midi, à peine rentré à la maison de la messe dominicale, le père fait fièrement remarquer que "le curé a b'en prêché encore une fois " et que "ils" finiront bien un jour ou l'autre " par en faire un monseigneur". Sur cette lancée, la conversation s'engage sur le contenu de la cérémonie à laquelle la famille vient d'assister. Puis, juste avant que les plats ne soient servis, Caleb demande à Émilie de prononcer le Bénédicité au nom de tous.

Petit bond dans la diégèse : Émilie est devenue institutrice et l'un des ses élèves, Lazare Pronovost (Michel Goyette), subit une crise d'épilepsie en pleine classe (épisode 3). Le lendemain, Émilie demande aux enfants de prier pour que le garçon "puisse guérir de sa maladie et revenir en classe le plus vite possible ». Le geste n'a rien de spontané et son but n'est pas de donner de la force aux jeunes, effrayés par ce qu'ils ont vu, puisque c'est Lazare, et non eux, qui fait l'objet de la prière. Par cette séquence, la narration s'assure simplement, une fois de plus, d'illustrer la présence du religieux dans les comportements quotidiens.

À la manière de vignettes illustrant la vie et les mœurs d'autrefois, ces exemples nous montrent un catholicisme pé- 
nétrant les multiples facettes de la culture quotidienne. La religion trouve sa place en toutes circonstances, qu'il s'agisse d'imposer la discipline, de faire sens d'un désarroi ou encore d'animer les échanges à table. Cela dit, en elles-mêmes, ces quelques scènes demeurent anecdotiques. Leur seule fonction consiste à permettre aux téléspectateurs de 1990 d'humer un certain parfum ${ }^{10}$. Elles participent d'une recherche méticuleuse de vraisemblance par les détails, ce que Roland Barthes nomme l'effet de réel. Le récit dépeint la maisonnée de Caleb Bordeleau comme un endroit où la religion s'avère prégnante, mais il n'en reste rien passées ces premières séquences qui remplissent à peu près la moitié de l'épisode. Dans la suite de la télésérie, le discours et le comportement de Caleb ne laissent jamais voir, chez lui, un quelconque investissement dans la religion. Au-delà de ce premier épisode, le père ne se réfère jamais aux enseignements religieux qu'il a reçus. Quant à Émilie, qui devrait en toute logique être influencée dans ses attitudes par son éducation familiale, jamais ne la verra-t-on, plus en avant dans le récit, manifester le moindre désir de perpétuer au sein de son propre foyer la foi ou la culture religieuses de son père.

Le second épisode nous montre comment la religion renforce la cohésion sociale. Dès la toute première scène, Dosithée Pronovost (Pierre Curzi), le père de Lazare, recommande à Émilie, sur qui circulent des bruits de reproches au village, d'aller se présenter au curé pour le gagner à sa cause et sa personne, et ainsi faciliter son intégration au sein de la communauté de Saint-Tite. «Je me disais : allez donc en parler au curé ; c'est un bon prêtre p'is y vous connaît pas beaucoup, explique-t-il à la jeune femme. Ça prendrait juste une couple de mots en chaire un bon dimanche, $p$ 'is tous les critiqueux rentreraient sous terre " (épisode 2). Un peu plus tard, à la messe dominicale, le curé donne ses consignes aux 
paroissiens sur le comportement à adopter envers le projet d'Émilie de monter avec ses élèves, dans leur école, une séance sur la Nativité pour le divertissement des parents. «Ce serait la charité chrétienne de l'aider un peu ", décrète-t-il avant d'ajouter, un brin condescendant: «Et puis... si elle manque son coup... de ne pas trop rire d'elle ». Le curé sert de guide, de conseiller, capable par ses mots d'orienter les comportements collectifs ou de rassembler les gens autour d'un projet.

L'autorité du prêtre se vérifie encore au soir de la séance (id.). D'une part, il a droit à la place d'honneur dans la petite école. La chaise de l'institutrice, ersatz de fauteuil magistral, lui a été réservée et mise bien en évidence au premier rang. Tous les paroissiens déjà arrivés l'accueillent avec déférence tandis qu'il se rend à sa place. Son statut lui attire également des salutations distinctes au moment de souhaiter la bienvenue au public. D'autre part, c'est en vertu de son autorité reconnue que le curé peut prétendre parler au nom du groupe à la fin de la séance qui a fait un triomphe. "Je crois pouvoir me faire l'interprète de tous en proclamant combien le spectacle qui vient de nous être offert nous a réjoui le cœur", lance-t-il d'abord à l'intention d'Émilie et de sa troupe. « Soyez assurés que tous, nous formulons des vœux encore plus ardents à votre intention et que nous vous remercions du fond du cœur », ajoute-t-il un instant plus tard. Ici, la figure ecclésiastique incarne la collectivité, car le curé rassemble autour de sa personne les villageois dont les voix empruntent la sienne. Il n'y a donc qu'un seul message livré, qu'une seule opinion émise ${ }^{11}$.

L'obtention d'un soutien moral et psychologique constitue le troisième angle sous lequel le religieux pénètre la vie des villageois. Encore une fois, la télésérie se charge de l'inscrire assez tôt dans le récit. 
Dans un premier temps, les séquences consacrées à la maladie d'Ovide (Patrick Goyette), un autre des garçons Pronovost, présentent la dévotion comme une disposition sincère, engagée et engageante (épisode 5). Avec tendresse, le père porte un crucifix aux lèvres du malade; avec piété, la mère récite un chapelet pour le salut de son fils ; avec ferveur, les frères et les sœurs prient ensemble. La religion et la foi catholiques agissent comme pilier soutenant les défaillants dans l'épreuve. Tandis que la séquence où Émilie faisait prier ses élèves pour Lazare ne laissait apparaître aucune émotion particulière dans la petite école, celle-ci nous montre des êtres éplorés, touchés au cœur par les événements et puisant dans leur foi de quoi résister à la tourmente qui secoue la famille.

Par contre, vers la fin de la séquence, un court dialogue entre Dosithée Pronovost et deux de ses enfants vient abruptement jeter une lumière différente sur la pratique religieuse et tout ce qui l'entoure :

- Possible que la fièvre tombe à soir, annonce le père d'une voix qui se veut rassurante. Voulez-vous faire une prière?

- On vient de dire un chapelet, précise Lazare.

- Ah! B'en... correct, d'abord.

— On peut b'en dire tout un rosaire, suggère sa sœur Rosée (Isabelle Rosa). Ça peut pas nuire.

À l'encontre de ce que le début de la séquence donnait à voir, la religion apparaît soudainement comme une opération mécanique et rationalisée. Une activité qu'on pratique en suivant le manuel d'instruction. C'est le rituel prescrit, bien plus que la conviction ou la foi, qui guide désormais les gestes. Si Rosée propose de dire un rosaire entier, ce n'est pas parce qu'elle en ressent intérieurement le besoin, mais simplement parce que, apparemment, «ça peut pas nuire » à son frère. 
Le dernier angle sous lequel Les Filles de Caleb aborde la religion se rapporte à la représentation de l'Église catholique et de ses représentants. Le curé, d'abord condescendant et vaguement méprisant avec Émilie, ne devient sympathique qu'après l'enracinement social de la jeune femme. On décèle donc, dans le portrait du prêtre, une critique de l'opportunisme historique de l'Église, récupérant un jour ce qu'elle avait condamné la veille - depuis les superstitions paysannes dans les campagnes européennes du Moyen âge jusqu'aux organisations syndicales des villes industrielles occidentales du XX siècle - ou pactisant afin de se coller aux gagnants. Devant les souffrances endurées par Félicitée Pronovost (Véronique Le Flaguais), clouée au lit par un accouchement cauchemardesque (épisode 7), le médecin lui-même, pourtant membre de la petite élite locale avec le curé, ne peut retenir une tirade anticléricale: "Enfanter dans la douleur!, s'exclame-t-il sur un ton de révolte. Facile à dire, ça, pour l'Église... Mais vient un temps que c'est plus possible. [...] Même un médecin en peut plus de faire souffrir son monde. » Préférant la vie d'un poupon à celle de sa mère, le curé signifie que l'Église se soucie bien peu de ceux qui restent, comme si la vie abstraite comptait plus à ses yeux que les vivants ou que le concept valait plus que la chose.

Les passages que nous venons de présenter surviennent assez tôt dans la narration parce que leur tâche première consiste à faire naitre une atmosphère. Puisque la télésérie cherche le vraisemblable et que la mémoire collective se souvient d'une religion catholique autrefois envahissante, imprégnant tout et tous, il tombe sous le sens d'affirmer catégoriquement la prégnance du religieux dès le départ. Mais une fois cela fait, le récit n'y revient plus. Peut-être parut-il plus prudent à l'équipe de production derrière Les Filles de Caleb de ne pas pousser plus loin dans cette veine? En effet, des protagonis- 
tes se référant sans cesse aux Dix Commandements ou aux paraboles bibliques risquaient de s'aliéner un auditoire contemporain, incapable de s'identifier à eux ${ }^{12}$. Caleb Bordeleau n'est donc un homme religieux que pendant une moitié d'épisode.

L'accumulation des séquences crée l'ambiance et pose un cadre de vie. À l'intérieur de ce dernier, par contre, les villageois manifestent une évidente capacité de faire la part des choses et de distinguer les préceptes cléricaux des exigences de l'existence ${ }^{13}$. Allons plus loin : aucun protagoniste des Filles de Caleb n'est véritablement religieux. Aucun ne vit sa foi quotidienne avec ferveur. Berthe Auclair (Nathalie Mallette), l'amie d'Émilie qui a choisi de se faire moniale, n'est même pas certaine de croire. Félicitée évoque tout le temps le bon Dieu, mais elle ne prie à peu près jamais même si le malheur s'acharne sur sa famille. Au fil de la télésérie, une unique réplique affirme timidement chez elle une certaine dévotion : à la toute fin du récit, devenue veuve, elle dit se réjouir d'aller habiter chez sa fille Éva (Ariane Frédérique) parce que "c'est moins loin de l'église. Il me semble que je vais être b'en, là-bas. » (épisode 20) Durant l'épisode initial, au retour de la messe, tous les Bordeleau rient de bon cœur du fait que Caleb a sommeillé durant la cérémonie. C'est qu'ils sont pratiquants, les Bordeleau, mais pas fanatiques. Plus tard, c'est Dosithée et trois autres villageois qu'on voit s'amuser à se remémorer le temps de leur folle jeunesse, lorsque l'envie leur prenait de rire un peu aux dépens du curé (épisode 3).

En somme, si la société rurale canadienne-française nous est montrée comme fortement marquée par l'encadrement de la religion catholique, les protagonistes du récit en demeurent néanmoins, dans l'ensemble, passablement détachés. Le souci de vraisemblance impose à la narration un mi- 
nimum de contraintes, au-delà duquel elle refuse manifestement d'aller.

\section{La famille}

Pour l'essentiel, les familles Bordeleau et Pronovost donnent vie à une série de clichés. Leur mode de vie et leurs manières d'être se conjuguent pour donner corps à certaines des représentations stéréotypées qui ont contribué à renforcer l'idée d'un particularisme canadien-français.

Première caractéristique, ces familles sont nombreuses. Conformes, donc, aux attentes des mémoires contemporaines. Les Bordeleau ont sept enfants et les Pronovost neuf. Émilie et Ovila, quand ils fondent leur propre famille, reproduisent ce modèle en concevant dix enfants. De ce point de vue, le couple donne l'impression d'évoluer dans un monde au sein duquel les générations nouvelles recréent sans cesse les modèles d'antan, un monde qui se régénère lui-même avec une mécanique implacable, comme au pays de Maria Chapdelaine où rien ne doit mourir ni changer. L'importance dramatique de la famille est néanmoins inversement proportionnelle à sa taille. Aucun rôle spécifique ne lui est attribué dans le développement de la diégèse. La grosse famille existe pour qu'on la voie, sans plus, et sa simple existence suffit à perpétuer un mythe fort, intériorisé depuis longtemps par les Québécois d'héritage mémoriel canadien-français ${ }^{14}$.

Les familles vivent aussi dans l'harmonie. Le bonheur règne entre tous. Aucun véritable antagonisme ne perturbe les relations interpersonnelles tant chez les Bordeleau que chez les Pronovost. Chez les premiers, il y a bien Émilie qui se rebelle contre son père dans les toutes premières minutes de la télésérie, mais la séquence ne dure guère et surtout ne laisse aucune trace chez l'un comme chez l'autre. La vie paisible a donc tôt fait de reprendre son cours. Chez les seconds, malgré 
qu'il soit sorti perdant de sa lutte avec Ovila pour le cœur d'Émilie (épisodes 2 à 5), Ovide ne traîne aucun stigmate de sa déroute. Auprès de la belle, il manifeste une seule fois sa rancœur (épisode 5) ; auprès de son frère, jamais. Un vrai champion de l'abnégation. Un apôtre de la bonne entente infinie.

Le traitement de cet élément particulier du récit est une concession faite au mythe de la famille harmonieuse d'autrefois. Au temps de la rivalité fraternelle, on avait parfois vu Ovila bouder quand les choses n'évoluaient pas à son goût. Il s'agissait alors de montrer son immaturité, de cerner un peu mieux sa psychologie en prévision des développements futurs. Avec Ovide, ce genre d'exigence narrative n'existe pas. Son personnage n'a d'importance que le temps de sa rivalité avec Ovila. Dès que la maladie le contraint d'oublier Émilie, il devient insignifiant et, à ce titre, sans intérêt pour la suite du récit. Cela étant dit, du point de vue du discours narratif sur la famille, Ovide ne peut pas s'effacer en douce et, comme ses frères Edmond et Émile, dire parfois la moitié d'une réplique dans le reste de la télésérie, car s'il fallait que les choses se passent ainsi, le maintien de rapports hostiles avec son frère et sa belle-sœur resterait un possible narratif dans l'esprit du téléspectateur. Pour prouver que l'harmonie familiale existe encore et toujours chez les Pronovost, il faut donc aller plus loin et montrer le garçon explicitement engagé dans la dynamique familiale. C'est d'ailleurs précisément ce qui se produit : Ovide, sincèrement heureux, est le plus festif de tous le soir des fiançailles de son frère avec Émilie (épisode 10) ${ }^{15}$.

L'harmonie familiale se devine aussi dans certaines images de transition entre séquences, par exemple celles de l'épisode 4 nous montrant des villageois qui travaillent ensemble aux champs : les uns coupent le foin, les autres le ramassent à la fourche, et d'autres encore, debout dans les charrettes, l'y entassent. Ce n'est pas la version paysanne d'un 
taylorisme aliénant que ce travail à la chaîne illustre, mais bien l'entraide humaine, le sens du bien commun, l'esprit de groupe. Sur fond de musique guillerette, des habitants canadiens-français sont occupés à prospérer ensemble dans la bonne humeur. Ces scènes bucoliques n'apportent rien au déroulement du récit, mais elles servent à compléter le tableau de mœurs.

Fait à noter, la représentation des rapports harmonieux au sein de la cellule familiale est à peu près complètement dénuée de substance. Sur ce plan, comme sur celui de leur taille, les familles n'ont d'autre fonction que servir de repère immédiat et superficiel ; leur représentation mise sur un cliché qui vise à susciter un sentiment de familiarité. Émilie, par exemple, n'entretient pas de relations affectives développées avec ses frères et sœurs, pas plus qu'avec sa mère. Si elle manifeste beaucoup d'attachement à son frère Napoléon dans certaines scènes des premiers épisodes, l'âge du garçon environ sept ans - ne favorise pas une grande complicité entre eux. Leurs rapports en restent au niveau des blagues et des banalités. Elle échange ici et là quelques mots avec sa sœur Année qui est juste un peu plus jeune qu'elle, mais les deux n'ont pas de conversations à proprement parler et rien ne témoigne d'un attachement de l'une pour l'autre. Il en va de même pour Célina, la mère, avec laquelle Émilie ne converse jamais en tête-à-tête. Les frères et sœurs Bordeleau disparaissent complètement du récit après le mariage d'Émilie et celle-ci ne prend jamais de leurs nouvelles. Elle écrit assez régulièrement à son amie Berthe pour chercher conseil ou s'épancher sur son sort, mais pas à ses parents. Caleb est le seul avec lequel Émilie discute à l'occasion à cœur ouvert. C'est lui qui aborde la question de la vie sentimentale de sa fille et se préoccupe de savoir si elle est heureuse ou si elle a choisi un bon parti. C'est aussi lui, et non pas Célina, qui 
entreprend Émilie pour la préparer à sa vie de femme mariée, une tâche que la tradition réserve pourtant à la mère. De tous les membres de la famille Bordeleau, Caleb est bien le seul, outre Émilie, à posséder une existence propre et autonome.

Les rapports interpersonnels et intergénérationnels sont plus développés au sein de la famille Pronovost. Certaines scènes nous donnent à voir les enfants jouant, discutant ou se chamaillant ensemble. Émilie fraternise d'abord avec Ovide, l'aîné des garçons, puis avec Rosée, l'aînée des filles, puis avec Lazare, et puis Éva... Et, bien sûr, avec Ovila. Là encore cependant, les contacts entre l'institutrice et ses beauxfrères et belles-sœurs n'acquièrent jamais de substance véritable. Rosée disparaît du récit dès son mariage. Éva n'assume un rôle de compagne et d'amie qu'après que sa sœur ait quitté la maison familiale, mais rien de très profond ne se développe entre Émilie et elle. Leurs conversations, banales, se déroulent chaque fois en présence d'une tierce personne.

Partout visibles dans Les Filles de Caleb, les marques de l'encadrement familial n'en restent pas moins remarquablement peu contraignantes. L'omniprésence de la famille n'empêche pas le récit de se déployer autour de la vie d'un protagoniste en particulier, Émilie Bordeleau. En ce sens, il s'avère foncièrement individualiste, à l'image des valeurs les plus préconisées dans le Québec contemporain. Tandis que l'omniprésence de la famille favorise, chez le téléspectateur, le repli sur une mémoire heureuse du Canada français, une mémoire capable de faire quelque peu contrepoids aux solitudes et aux désarrois d'une société urbaine atomisée, son insignifiance narrative fait en sorte que rien ne vient remettre en question les gains que la conquête de la liberté individuelle a procurés à chacun. 


\section{Le terroir}

La campagne constitue tout l'horizon des pères Bordeleau et Pronovost. Tant Caleb que Dosithée s'étonnent, chacun son tour, qu'un jeune homme comme Ovila puisse ne manifester aucun intérêt pour la vie de cultivateur (épisode 4). Le grand souci de Dosithée, c'est de trouver, parmi ses garçons, quelqu'un pour lui succéder sur la ferme. Lazare et Ovide, l'un à cause de l'épilepsie et l'autre de la tuberculose, sont hors concours. Émile est plutôt chétif. Edmond, Ovila, Oscar et Télesphore ont la constitution physique et la santé adaptées aux durs labours, mais le "bien» ne les intéresse pas. Leur horizon de vie répond aux profonds bouleversements socio-économiques qui orientent le développement du Québec à cette époque, alors que la ville et le salariat livrent une concurrence de plus en plus soutenue à la campagne.

L'aisance des agriculteurs des Filles de Caleb est pourtant indéniable. Aisance toute relative et qu'il faut remettre dans son contexte, certes - personne ne roule sur l'or et les bâtiments de ferme, entre autres, auraient besoin d'un coup de pinceau -, mais tout de même suffisante pour que l'indigence ne soit pas crainte. Dosithée déplore que «les habitants ont b'en du trouble aujourd'hui » (épisode 8), il se plaint " d'en arracher avec la misère qui arrête pas de rôder partout alentour» (épisode 9), mais rien de ce qu'on voit au fil des épisodes ne permet de conclure qu'il vit lui-même des soucis pécuniaires. Au début du récit, par exemple, il spécule sur les possibilités d'obtenir une concession de bois (épisode 2). Son projet se concrétise, de telle sorte que par la suite, lorsqu'il part bûcher durant les mois d'hiver, c'est pour son propre profit et non pour celui d'une papetière. Autre exemple : un jour où Émilie et Ovila, nouvellement mariés, constatent leur peu de moyens comparativement à Dosithée, ils évoquent la possibilité de devoir " piger dans le caveau des parents pour man- 
ger jusqu'à la récolte » (épisode 12), sûrs qu'ils sont d'y trouver des réserves suffisantes. Enfin, Dosithée laisse un important héritage en terres et en argent (épisode 17). Pour sa part, Caleb se voit contraint d'abattre son étalon après que la bête s'est cassée les pattes (épisode 1), mais cela ne représente pas pour lui une catastrophe financière. Et juste avant qu'Émilie ne reparte pour sa dernière année d'enseignement, il lui offre une pouliche et une calèche qu'il a récemment achetée " par adon, il y a une couple de semaines ", un cadeau considérable dont la valeur n’échappe pas à sa bénéficiaire : " Mais qu'estce qui vous a pris, papa ?! Ça a quasiment pas de bon sens, une fille de vingt ans qui a déjà sa calèche à elle toute seule !» (épisode 10).

Dans Les Filles de Caleb, la ruralité ne produit pas des bienfaits sur la seule situation matérielle des cultivateurs. Elle contribue aussi à la pureté du cœur et de l'âme. Aucune immoralité ne semble avoir cours à Saint-Tite ni à Saint-Stanislas ${ }^{16}$. Pas de comportements vulgaires, pas de jurons lâchés sans vergogne. À l'Hôtel du Grand Nord, les hommes parlent de sexualité avec pudeur, sans oser nommer les choses ${ }^{17}$. Tous boivent dans la bonne humeur, mais personne ne franchit les limites de la décence qu'on se représente fixées par la morale publique ou l'Église catholique. Ces hommes qui, pour la plupart, vont besogner dans le bois à chaque hiver n'ont rapporté des chantiers forestiers aucune mauvaise habitude langagière. Les bûcherons parlent la même langue que l'institutrice.

Dosithée tire sur sa pipe en de rares occasions. Pour sa part, Caleb ne fume jamais, pas même dans ses soirées de détente (épisode 6). Le père Bordeleau reçoit pourtant en cadeau une pipe de la part du second prétendant de sa fille, Henri Douville, lorsque celui-ci se permet une visite surprise à la jeune femme (épisode 8 ). Le récit affirme ici qu'un objet 
lié au tabac constituait, à cette époque, un cadeau sans risque pour un homme qu'on ne connait pas et dont on ne sait rien des habitudes et des préférences. Ce faisant, il induit l'idée que le tabagisme représentait un mode de vie, une habitude enracinée dans les mœurs, une activité fréquente, naturelle et partagée par tous les hommes. Le récit se fonde sur des faits vérifiables - on a longtemps beaucoup fumé - et son message accentue la vraisemblance de la reconstitution du passé opéré dans la narration, mais il se contredit néanmoins, ailleurs, en prêtant à tous ces hommes une attitude de détachement très anachronique face au tabac.

Quelques mots pour terminer sur l'alcoolisme d'Ovila. Ce dernier aime lever le coude et ce sont seulement ses excès ou leurs conséquences sur son état physique (épisodes 14, 15 et 20) qui intéressent la narration, car chacun annonce un développement négatif sur la vie de son couple. Caleb, Dosithée et les autres hommes, par contre, ne boivent pas. Jamais n'ont-ils envie d'un petit verre de caribou pour se réchauffer. Jamais envie de picoler pour se détendre. Des maris modèles, quoi!

\section{L'bomogénéité et l'unité}

Le thème de l'homogénéité et de l'unité de la société rurale canadienne-française se décèle dans l'étroitesse du milieu de vie autant que dans la composition démographique du milieu de vie des protagonistes des Filles de Caleb.

Bien que le récit se déroule presque totalement à SaintTite et à Saint-Stanislas, il n'y a, pour ainsi dire, pas de villages à voir dans la télésérie. Les scènes tournées en extérieur, mises à part celles qui se déroulent chez les Bordeleau et les Pronovost ou à l'école, se situent généralement dans un espace dénué de bâtiments, par exemple une route, un champ, ou une berge. Saint-Tite et Saint-Stanislas n'existent que de 
manière nominale. On soupçonne leur présence, mais on ne les voit pas. On les imagine là, tout autour des personnages qui en parlent à l'occasion, mais ils demeurent insaisissables, immatériels. De Saint-Tite, outre la demeure des Pronovost et l'école, on aperçoit l'intérieur de l'église, la devanture et l'intérieur de l'Hôtel du Grand Nord, et, enfin, la façade enflammée du couvent voisin la nuit où il brûle. Rien d'autre. De Saint-Stanislas, on ne voit que la ferme Bordeleau ainsi que le perron et l'intérieur de l'église ${ }^{18}$. C'est tout. C'est peu. Il n'existe conséquemment aucune communauté. Les Bordeleau et les Pronovost n'ont aucune vie sociale et apparemment ni amis ni parenté dans leur village respectif. À l'exception notable de la séquence sur la petite saynète de Noël organisée par Émilie ${ }^{19}$, jamais ne voit-on de fête populaire ou religieuse à l'occasion de laquelle se rassembleraient les villageois. Une telle absence de vie communautaire dans la représentation générale du monde rural canadien-français ne favorise pas l'émergence d'un sentiment de diversité chez le téléspectateur, bien au contraire.

Dans le cas d'Émilie, cette absence d'interaction avec les villageois se révèle presque comique. À l'été 1896, se livrant au bilan de sa première année d'enseignement, Émilie tente d'expliquer son emballement à son amie Berthe. Notamment, explique-t-elle, il y a la question du prestige social qui vient avec le statut d'institutrice. "T'es quelqu'un dans la paroisse, fait-elle valoir. Comme le curé, le docteur ou bien le notaire. » (épisode 4) Sauf qu'Émilie ne sort jamais de chez elle, à moins que ce soit pour rendre visite aux Pronovost, ses voisins. Va-t-elle au magasin général à l'occasion ? Chez l'apothicaire? Au bureau de poste? A-t-elle une seule raison de se rendre au village? Il est bien possible qu'elle soit devenue "quelqu'un dans la paroisse » depuis qu'elle y fait la classe, 
mais le téléspectateur se voit forcé de la croire sur parole parce que la narration ne le lui prouve pas.

Le choix qui a été fait de concentrer le déroulement du récit dans un nombre très restreint d'espaces privés se traduit à l'écran par une histoire dans laquelle ce qu'on voit - la composition des familles, les relations entre leurs membres, la vie en ville, le métier d'institutrice, etc. - ne possède aucune variante. Chaque chose figure comme un absolu. Pour chacune d'entre elles, un seul modèle s'offre à l'appréciation des téléspectateurs. Comme aucune base de comparaison n'existe, aucune différence substantielle ne devient alors possible ni même pensable. Les Bordeleau et les Pronovost fonctionnent dans la narration comme des familles archétypales, incarnant le degré zéro de la vie rurale dans le Canada français du tournant du $\mathrm{XX}^{\mathrm{e}}$ siècle. Le modèle familial de ces deux familles, c'est en même temps le modèle familial de l'époque. Le récit enferme toutes les familles villageoises à l'intérieur d'une représentation unique. La partie se confond avec le tout. L'homogénéité et l'unité de la société rurale d'antan se voient confirmées.

Sur le plan ethnoculturel, les personnages composent un ensemble quasiment homogène. Il n'y a pas de couples d'ascendance mixte, aucun individu n'est métissé et personne, parmi les Canadiens français, ne porte un patronyme d'origine étrangère, irlandaise par exemple.

Les Amérindiens ne composent pas la figure de l'autre par laquelle les Canadiens français se définissent, ni un quelconque partenaire, ni non plus un ennemi. Ils sont anecdotiques, comme une discrète note de bas de page : ici, c'est Ovila qui rentre un jour des chantiers la besace pleine de cadeaux indiens pour Émilie et les enfants (épisode 16) ; là, c'est l'expression d'un folklore associant les accouchements au passage des "Sauvages» (épisodes 7 et 15). Quelques-uns des 
stéréotypes négatifs contemporains associés aux Amérindiens - fainéantise, alcoolisme, infantilisme - se décèlent aussi, encore que leur présence n'a aucune conséquence sur l'évolution du récit dans telle ou telle autre direction. On apprend ainsi, de la bouche d'Ovide, qu'Ovila est allé boire son chagrin « chez les Indiens " pendant des mois après avoir été blâmé par Émilie pour la mort d'une de leurs filles (épisode 15). Le même Ovila soulignera également, après que son père l'a déshérité : "Je pense qu'il a voulu me dire qu'à trente ans, je pouvais plus continuer à jouer à l'Indien. Que j'avais une femme p'is sept enfants, p'is qu'il fallait que je m'en occupe. » (épisode 18) Par la bouche d'Ovila, le récit affirme que a) les territoires autochtones sont là où on va boire, ou plus exactement là où on peut vivre en ne faisant rien d'autre que boire, et que b) on ne vit pas à l'indienne, on « joue » à faire l'Indien. L'indianité signifie le manque de sérieux, l'irresponsabilité. Impossible de s'occuper convenablement de sa famille sans se dissocier d'abord d'un type de comportement amérindien.

Négative, l'image des autochtones dans Les Filles de Caleb n'en demeure pas moins à peine esquissée. Trois ou quatre coups de crayon furtifs, sans plus. Sur le plan des émotions, la figure de l'Indien est vide. L'approche contraste nettement avec celle réservée à la figure de l'Anglais.

Très peu présent au fil du récit, l'Anglais fait surface dans une demi-douzaine de scènes, dont trois à peine où il participe à l'action (épisodes 13 et 20). Sa figure antagoniste n'en est pas moins porteuse de malheurs et de difficultés pour le couple formé par Émilie et Ovila. Du point de vue du thème de l'homogénéité et de l'unité, il faut souligner que les contacts entre des protagonistes canadiens-français et l'Anglais ne surviennent que très occasionnellement et, pour les premiers, ne génèrent jamais rien de positif. Alors que l'Indien, 
infantile et décadent, ne vaut pas la peine qu'on s'y intéresse, l'Anglais, lui, exige défiance ${ }^{20}$.

Par rapport au Canadien français, l'Indien et l'Anglais vivent dans des univers distincts, parlent une langue distincte, ont des coutumes distinctes, bref, sont distincts. Comme rien de positif n'émane de l'un ou de l'autre, un Grec ancien transplanté dans le Canada français des Filles de Caleb comme par magie - celle du "gars des vues ", par exemple — y reconnaitrait sans doute l'archétype du barbaros, de l'étranger qui, précisément parce qu'il est étranger, est sans allure.

\section{La France et les Frangais}

Comme les Amérindiens, les Français et leur patrie ne s'inscrivent dans la trame narrative qu'indirectement, à travers le discours que tiennent les personnages à leur sujet. Cependant, et c'est en cela qu'ils se distinguent le plus nettement des autochtones d'un point de vue sémiotique, ils renvoient à des signifiés dont la valeur morale ou culturelle est parfois positive.

Dans Les Filles de Caleb, tout ce qui touche à la France passe par Henri Douville qui incarne tour à tour la double figure du Français — le « bon » et le «maudit» — et celle du Canadien français / Québécois mentalement colonisé par la France et sa culture. Nous avons montré que la relation d'Henri avec la langue française se révélait en définitive structurante sur le plan identitaire. Ce qui nous intéresse maintenant, c'est de replacer la question linguistique dans le contexte plus large des perceptions générales de la France et des Français.

Comme un certain Français de l'imaginaire, Henri se révèle fin causeur, érudit, galant, distingué, en plus de posséder un accent pointu en porte-à-faux par rapport à celui de 
ses interlocuteurs. Dès sa toute première apparition (épisode 4), sa maitrise brillantissime des subtilités de la langue française ne manque pas d'impressionner et de le distinguer des autres personnages de la narration. La diction est parfaite, le discours fluide, le vocabulaire précis, le propos cohérent. C'est lumineux. Les mots s'écoulent avec un fort débit quand l'inspecteur ouvre la bouche. Des torrents de mots dont les musiques, comme autant de vagues sur de stoïques récifs, se fracassent sur les oreilles éberluées d'un auditoire aux habitudes langagières autrement plus rustiques. L'inspecteur n'a rien de l'homme canadien-français - traditionnellement un homme de peu de mots - et son éloquence n'est pas celle du conteur. Ce dernier parle beaucoup quand il raconte. Henri, lui, parle beaucoup quand il parle. Nuance.

Les modes d'expression de l'inspecteur, en gestes autant qu'en mots, sont à ce point étranger à l'univers de Caleb que celui-ci, la première fois qu'il le rencontre, en demeure perplexe : «Est-ce que vous êtes français, monsieur Douville ? ", lui demande-t-il directement (épisode 8). La suite de l'échange entre les deux hommes introduit dans la narration le stéréotype du Français plus cultivé et plus sophistiqué que le Québécois. "Je me disais qu'avec des manières de même, continue le père Bordeleau en référence au langage et au comportement d'Henri, vous devez être quelqu'un de b'en connaissant. » (id.) Dans son esprit, les signifiants " raffiné », induit par son commentaire sur les belles manières de l'inspecteur, et " érudit", ou connaissant, se raccordent tous deux au signifié «français ». Caleb ne dit pas à Henri - ce qui ferait pourtant sens compte tenu de sa question initiale — qu'avec "des manières de même, vous devez être français ». Il lui dit plutôt que les manières de l'inspecteur, qu'il imagine françaises, lui confèrent une allure savante. Dans la tête du père Bordeleau, Henri, puisqu'il s'exprime si bien, doit forcément 
venir de France. L'érudition, le raffinement et les Français sont intimement liés dans son esprit. L'un ne se pense même pas sans les autres. Etrre raffiné, pense Caleb, c'est être français. Et être français, c'est être érudit. Les Français sont de fins savants. Tout est dans tout. Logique pernicieuse, qui lève sur le voile sur l'existence de complexes d'infériorité que lès Québécois d'héritage canadien-français ressentent parfois face à la France.

Henri lui-même possède ce genre de complexes. Le temps d'une scène, il personnifie l'archétype du Canadien français colonisé par la France, sa culture, son souvenir, son « esprit », et ainsi de suite. La scène se déroule à Saint-Stanislas, le 24 décembre 1898. Henri et les Bordeleau s'apprêtent à se rendre à l'église pour la messe de minuit, au cours de laquelle il est prévu qu'Émilie et lui consacrent leurs fiançailles. Au cours d'un échange de mondanités, la jeune femme entend son promis mentionner "la métropole » et lui demande candidement s'il parle de Montréal. Henri, en bon petit colonial impénitent, est stupéfait de la question :

Non, Émilie... La France! Vous savez bien que les gens des colonies réservent le terme «métropole » pour la France elle-même. [...] Dans le cœur des Canadiens français, la France sera toujours notre mère. Et nous serons toujours des Français. Nous parlons la langue des Français. Nous avons le code Napoléon. Et nous sommes entêtés... Comme les Normands, nos ancêtres. (épisode 9)

L'inspecteur professe envers sa " mère patrie » une soumission volontaire. Henri n'est pas un esprit indépendant, il voit petit, il se complait dans son statut d'éternel subordonné (« les gens des colonies»). La France est grande, je suis minuscule et c'est ma joie. La tirade d'Henri illustre une sorte de pathos misérabiliste. Elle s'inscrit dans la tradition du mé- 
pris de soi diagnostiquée par toute une succession d'observateurs du Canada français et du Québec francophone. Ce n'est pas la France qui se trouve ici mise en cause, mais les attitudes historiques des Canadiens français à son égard.

Au total, et malgré qu'ils aient recours à bien des stéréotypes, les six thèmes analysés dans les pages précédentes composent un agrégat équilibré. Globalement nuancée, la représentation de la francité laissait deviner l'exiguïté du milieu de vie, le poids parfois pesant d'une certaine "tradition ", la méfiance et les complexes envers les corps étrangers, mais aussi un véritable sens de la famille, une solidarité dans l'action et un lieu de partage à la fois matériel et émotionnel. La télésérie a ainsi rappelé que le passage à la modernité avait causé sa part de dommages collatéraux. L'effort investi par l'équipe de production pour parvenir à nuancer son discours sur le passé a porté ses fruits : les téléspectateurs ont pu se complaire dans une douce nostalgie du bon vieux temps sans verser dans un passéisme ridicule, comme ils ont pu se mirer dans leur écran de télévision sans s'y trouver déformés.

\section{L'éruption d'une mémoire d'Émilie Bordeleau}

L'histoire originale des Filles de Caleb plonge ses racines dans le réel. Émilie Bordeleau et Ovila Pronovost furent en effet les grands-parents maternels de celle qui les a d'abord révélés au grand public par un roman, Arlette Cousture. Plusieurs personnages secondaires ont eux aussi existé, comme sont authentiques les lieux où se déroule l'essentiel du récit. Toutefois, c'est bel et bien à une fiction que nous avons affaire. Arlette Cousture n'a pas tâché de narrer la vie d'Émilie et d'Ovila avec le plus d'exactitude historique possible. La romancière ne s'est pas efforcée de faire œuvre d'historienne ni de restituer le passé avec un maximum de fidélité, préférant plutôt s'inspirer librement de deux personnes réelles - 
mais qu'elle n'a jamais connues - pour fabriquer des personnages et leur faire vivre un passé inventé. De son propre aveu, elle n'a pas dépeint ses grands-parents tels qu'ils furent, mais plutôt tels qu'elle a eu envie de se les représenter ${ }^{21}$. De son côté, le scénariste Fernand Dansereau s'est efforcé de rendre le plus fidèlement possible le roman dans une écriture adaptée aux particularités du médium télévisuel ${ }^{22}$. C'est donc dire que la dimension fictive du récit original n'était aucunement diminuée dans le scénario que nous avons analysé.

\section{Percheron superstar}

Du sentiment d'authenticité historique que dégage la télésérie, il faut bien dire qu'il ne naquit pas sans raison. $\mathrm{La}$ reconstitution de l'environnement matériel de la campagne canadienne-française du début du $\mathrm{XX}^{\mathrm{e}}$ siècle, minutieuse et professionnelle, s'avéra remarquable à tous points de vue. En fait, en ont conclu d'aucuns, c'était tellement réaliste que ça ne pouvait que traduire le réel, tellement vraisemblable qu'il fallait que ce soit vrai... Connu du public, le lien de parenté unissant Arlette Cousture aux familles Bordeleau et Pronovost ne fit qu'ajouter à l'impression d'authenticité historique en confirmant que les principaux protagonistes à l'écran avaient une origine historique. Or si ceux-ci avaient vraiment vécu, et si le monde matériel des Filles de Caleb était à ce point authentique, ne s'ensuivait-il pas, logiquement, que les personnages l'étaient tout autant? Qu'ils furent en 1910 tels qu'ils étaient représentés en 1990 ? C'est une conclusion à laquelle sont parvenus plusieurs téléspectateurs, parfois aidés en ce sens, il faut le dire, par des médias comme Le Journal de Québec qui, en novembre 1990, titrait en grosses lettres de couleur, à la une de son cahier "Supplément du samedi, que l'acteur « Roy Dupuis et Ovila Pronovost ne font qu'un ${ }^{23} »$. 
Des journalistes ont aussi avalisé la thèse de l'authenticité historique. « Je pensais à ma marraine Blandine, à ma mère qui ont enseigné elles aussi en Mauricie dans un village pas loin de Saint-Tite et Saint-Stanislas, écrivit par exemple Ghislaine Rheault, du Soleil, au lendemain d'un visionnement promotionnel offert à la presse. Et qui nous racontaient l'eau gelée dans l'école, le poêle qu'il fallait chauffer, le bois qu'il fallait rentrer ${ }^{24}$ ", et tant d'autres choses encore que montre la télésérie. Son collègue Daniel Rioux, dans Le Journal de Québec, laboura le même sillon quand il remarqua que Caleb Bordeleau composait « un attachant personnage qui nous rappelle qu'il y avait des pères de famille très présents auprès de leurs enfants, dans les années $1890^{25}$ ».

Du point de vue de l'authenticité nationale, par contre, ce que suscita Les Filles de Caleb fut d'un tout autre ordre. La grande force de l'œuvre, dans cette optique-ci, ne fut pas didactique, mais identitaire. C'est qu'en plus de représenter les anciens modes de vie, Les Filles de Caleb permit aussi aux téléspectateurs - cela, beaucoup l'affirmèrent sans détours de regarder ce que " nous » étions - et sommes encore " nous» les Québécois d'héritage canadien-français. Suivre fidèlement cette émission, dans cet esprit, c'était se donner les outils pour se comprendre un peu mieux individuellement et collectivement.

Plusieurs témoignages allèrent en ce sens. «J'ai reçu des lettres de femmes me disant qu'elles plantaient leurs enfants devant le petit écran pour qu'ils ne ratent pas l'émission, raconta par exemple Louise Cousineau, la chroniqueuse de télévision du quotidien montréalais La Presse. D'autres leur accordaient une permission spéciale de rester debout [...], car elles voulaient que leurs enfants puissent voir ce téléroman qui relate "notre" histoire ${ }^{26}$. . Ailleurs, dans une sorte de postmortem publié en mars 1991 dans un magazine populaire, le 
journaliste Germain Monté vanta la qualité exceptionnelle d'une cuvre "servi[e] dans un cadre authentiquement québécois ${ }^{27}$ », supérieure à tout ce qui s'était fait jusqu'ici en matière de télévision en raison de «ce reflet presque parfait de nous-mêmes qu'elle nous renvoyait, chaque semaine, inlassablement, indomptablement, comme le reflux de la mer sur le rocher Percéé ${ }^{28}$ ». Cette œuvre, c'était tellement « nous » qu'au temps de la diffusion de la télésérie, s'il faut en croire Monté, des gens seraient même allés jusqu'à soutenir sur des tribunes téléphoniques à la radio que «tout bon Québécois fier de sa langue et de son pays [le Québec, de toute évidence] devait se faire une obligation morale de regarder Les Filles de Caleb $^{29} \%$.

Les opinions de ce genre ne furent pas l'apanage des publications commerciales. À preuve, les passages suivants consacrés aux Filles de Caleb sur un site Internet dédié à Roy Dupuis. « [Jean] Beaudin en réalisant cette série nous a fait le plus beau cadeau qu'on puisse faire à un peuple, écrivit la webmestre. Un portrait en héritage. Tout y est. C'est un miroir parfait. Ça donne le vertige. C'est tellement nous. Cette terre est combien nôtre. On la sent $t^{30}$. Un peu plus loin, sur une note davantage personnelle, elle ajouta : «L'histoire commence au début des années 1890 alors qu'Émilie entre dans l'adolescence. Mes grands-parents sont nés à la fin de cette décennie. Ce passé m’appartient et ne m’apparaît pas si éloigné $^{31}$.»

On ne se surprendra pas qu'en contexte d'affrontement Québec-Canada, comme c'était le cas en 1990 et 1991, l'authenticité nationale inscrite au cœur des Filles de Caleb servit même de référence politique. Denis Massicotte, dans le quotidien Le Soleil, cita ainsi la télésérie pour asséner un argument massue à Clyde Wells, le premier ministre de TerreNeuve, "qui ne croit pas que les Québécois sont distincts ${ }^{32}$ ». 
À l'été 1991, un article de la Presse canadienne révéla qu'un citoyen, fabriquant de monuments funéraires de son état, venait d'offrir une de ses pierres tombales à la mémoire d'Ovila Pronovost ${ }^{33}$. C'est qu'en visitant le cimetière de SaintTite, l'homme, qui souhaitait s'arrêter sur la tombe d'Ovila, avait eu la déception de constater que plus rien n'indiquait l'emplacement où ce dernier avait été mis en terre quarante ans plus tôt. De retour chez lui, il décida donc de remédier à la situation en faisant ériger à ses frais un monument, rien de moins. Étrangement, à côté du nom d'Ovila, il y grava aussi celui d'Émilie Bordeleau, pourtant inhumée à Saint-Stanislas, de façon à ce que le couple soit réuni dans l'éternité malgré tout. L'année suivante, la même agence de presse nous apprit que des touristes en excursion au lac Perchaude, à proximité des terres qui appartenaient autrefois à Dosithée Pronovost, allaient jusqu'à recueillir, pour emporter, un peu d'eau du lac ou un peu de terre de ses berges, copeaux d'une histoire et fragments d'un passé on ne peut plus authentique ${ }^{34}$.

Toutes les anecdotes qu'ont publiées les journaux en rapport avec la popularité massive des Filles de Caleb n'avaient pas la même portée, tant s'en faut. Mais même l'apparente insignifiance peut en dire long sur l'attrait que la télésérie a continué d'exercer. Qu'on en juge : des années après la diffusion, les quotidiens La Presse et Le Soleil prirent la peine de rapporter le décès de tel ou tel autre enfant de la véritable Émilie Bordeleau ${ }^{35}$ et même celui du décorateur qui avait travaillé sur le plateau de tournage des Filles de Caleb ${ }^{36}$. Et surtout, que penser du fait qu'un journaliste, en 1994, crut bon d'informer ses lecteurs que dans tel verger ouvert au public, le gros percheron noir qui conduisait les visiteurs jusqu'aux pommiers était "nul autre que le même animal qui a "joué" dans la série des Filles de Caleb, dans le rôle [!] de la monture du père $d^{\prime}$ Émilie $^{37}$ ? » 


\section{Le réel, la fiction et le diable}

Comme nous l'avons montré plus haut en examinant la représentation du pôle culturel de la francité, le récit fictif des Filles de Caleb était enrobé d'assez de véracité et de réalisme pour qu'un public peu ferré en histoire du Québec croie y retrouver une matière connue, même confusément, et sente sa mémoire sollicitée. Dès lors que les vecteurs de l'effet de réel - cette impression de réalisme née d'une accumulation de détails - se trouvent en nombres suffisants, une fiction historique bien menée peut réussir à tromper et convaincre qu'elle est le réel. Après tout, le diable ne loge-t-il pas toujours dans les détails?

En dépit du vernis de véracité plaqué sur son récit fictif, Arlette Cousture a eu bien des difficultés à s'expliquer l'adulation dont ont fait l'objet aussi bien les personnages et l'univers qu'elle a créés, que les personnes et les lieux réels qui les ont inspirés. "Peut-être, hasarda-t-elle un jour en entrevue, leur [les téléspectateurs] ai-je redonné une moitié d'eux-mêmes : l'envers de l'histoire, revue et corrigée par les cols romains, qu'on leur avait apprise. Mais en ce qui me concerne, ma grande difficulté a toujours été de faire comprendre que je n'avais pas écrit une biographie. C'est pas parce qu'on met un fait historique quelque part qu'on fait de l'histoire ${ }^{38}$. » En effet.

Et pourtant, comme on y a cru, à cette histoire! La frontière séparant le vrai du vraisemblable est toujours mince et fuyante, et, dans le cas des Filles de Caleb, le diable n'eut aucune difficulté à séduire le public et à l'inciter à la franchir. Certains téléspectateurs résistèrent, mais d'autres, au contraire, ne demandèrent pas mieux que de faire le pas décisif, petit pour eux, mais énorme pour la naissance et le déploiement d'un mythe. De ce pas découla une confusion souvent très grande entre le réel et la fiction, le passé et l'imagination, 
la vie et l'écriture. On honora la mémoire d'une personne en ayant le personnage en tête. On parla de la première comme si elle avait été le second. Franchir la ligne entre le réel et l'imaginé, c'était aussi la faire disparaître.

Des voyages en groupe ont ainsi été organisés pour permettre aux téléspectateurs de se rendre dans ces endroits de la Mauricie qui ont inspiré Les Filles de Caleb. Tout l'été 1991, des visiteurs de passage au cimetière de Saint-Stanislas ont déposé des fleurs en permanence sur la tombe d’Émilie Bordeleau $^{39}$. Ce genre de confusion était le même que celui à l'origine du don d'un monument funéraire à la mémoire d'Ovila Pronovost. "J'ai la satisfaction personnelle d'avoir rendu un hommage à cette famille", avait alors affirmé le donateur en commentant son geste de générositét ${ }^{40}$. La question se pose pourtant : à qui rendait-il réellement hommage en faisant ce don : à la véritable famille Bordeleau-Pronovost, dont il ne savait rien de plus que ce que la télésérie et le roman avaient pu lui transmettre, ou aux personnages fictifs du tandem Cousture-Dansereau qu'il avait appris à connaitre et à apprécier ? Quelques mois plus tôt, la municipalité de SaintStanislas avait annoncé sa décision de baptiser sa bibliothèque publique du nom d'Émilie-Bordeleau « en reconnaissance d'Arlette Cousture pour nous avoir fait connaître sa grandmère ${ }^{41} "$, aux dires d'un édile. Singulier hommage, qui n'est pas sans rappeler en apparence celui que la très sérieuse Royal Society of Chemistry de Londres rendit à l'automne $2002 \mathrm{au}$ détective Sherlock Holmes ${ }^{42}$. En effet, pour souligner l'influence exceptionnelle du personnage sur le développement de méthodes scientifiques dans la lutte au crime, la vénérable institution prit prétexte du centenaire de la publication de sa plus célèbre enquête, Le Chien des Baskerville, pour lui conférer le titre de membre honoraire. Mais c'est précisément là, dans ce geste d'honorer fondant la parenté des deux situa- 
tions, que s'est en même temps arrêtée la similarité des deux hommages rendus. Sherlock Holmes ne fut jamais qu'un personnage fictif ; il n'est pas un véritable détective demeuré toute sa vie inconnu, mais auquel un romancier doué aurait donné une seconde existence. S'il est bien possible et même probable qu'aux quatre coins du monde, des lecteurs confondent le réel et la fiction et s'imaginent que leur enquêteur favori a réellement vécu et solutionné tous ces mystères, il est clair en revanche qu'un embrouillement de la fiction et de la réalité n'a pas été à l'origine de l'hommage rendu au fin limier par la Royal Society. "Sherlock Holmes n'a bien sûr jamais existé, expliqua la directeur de la prestigieuse confrérie. [...] L'importance de sa légende, depuis des décennies, est néanmoins profonde [...]. Selon nous, le mythe de Sherlock Holmes est désormais tellement bien ancré dans la psyché nationale et internationale grâce aux livres, aux films, à la radio et à la télévision qu'il a presque transcendé les frontières de la fiction ${ }^{43}$."

Selon ce que nous pouvons déduire de ces marques de reconnaissance, l'Émilie Bordeleau honorée par ses admirateurs résulta d'une fusion de trois Émilie, l'humaine, la romanesque et la télévisuelle. D'un côté, les archives et l'existence de ses enfants et petits-enfants servaient à confirmer qu'elle avait bel et bien vécu à tel endroit et à telle époque ; de l'autre, le roman et la télésérie lui conféraient un visage, une voix, un tempérament, des ambitions, des rêves, des réussites et des échecs, bref, une existence autonome. Nous nous demandions plus haut à qui ces gens rendaient réellement hommage. $\mathrm{La}$ réponse est sans doute : à leur Émilie, celle qu'ils s'étaient inventée, celle à laquelle ils croyaient, donc celle qui était vraie pour eux. Une telle invention émana d'une profusion de détails réalistes que les esprits enregistrèrent et sur la base desquels ils accréditèrent le tableau d'ensemble. Ce fut l'effet 
de réel dans toute son efficacité. À moins qu'il ne s'agit de quelque diablerie ${ }^{44}$ ?

La revue de presse dont nous venons de rendre compte a à peine permis d'effleurer la question de la réaction populaire suscitée par la diffusion des Filles de Caleb. Par manque de données plus précises compilées à l'époque, il ne sera malheureusement jamais possible d'aller au fond des choses, comme il ne servirait à rien de sonder aujourd'hui ceux qui avaient regardé la télésérie sur la signification que cette émission avait prise pour eux au temps de sa diffusion. Tempus fugit, causant de ce fait bien des ravages et entrainant dans l'oubli de précieuses informations pour le chercheur intéressé par la mémoire. Les marques de reconnaissance d'une authenticité nationale et historique que d'aucuns accolèrent aux Filles de Caleb laissent seulement entrevoir la richesse de potentiel herméneutique qui, en d'autres circonstances, aurait pu s'offrir à nous. Si la confusion entre la chose et sa représentation a pu s'observer dans une série d'épisodes anecdotiques, cette confusion constitue en soi bien plus qu'une anecdote. Son analyse nous fait pénétrer à l'intérieur du fascinant mécanisme de construction a posteriori d'un réel. Elle nous rappelle ainsi la pertinence des récits de fiction pour l'historien. C'est déjà beaucoup.

\section{$* * *$}

La télésérie Les Filles de Caleb a beaucoup misé sur la francité pour instaurer un climat de connivence culturelle ou identitaire avec son public. Cela dit, l'impression d'authenticité qu'elle a laissée à son public et l'effervescence qui en a découlé ne s'expliquent pas totalement par la référence à l'héritage culturel français d'une majorité de Québécois, tant s'en faut. Il faut tenir compte de la production soignée de l'œuvre, de la promotion - formelle ou tenant du simple bouche-à- 
oreille des téléspectateurs -, de l'effet d'entraînement qui accompagne la mode du jour et du colossal succès de librairie des Filles de Caleb dans sa première incarnation, romanesque. Enfin, la télésérie avait beau fleurer bon la francité, son récit allait bien au-delà, d'une part en incorporant des références à la canadianité et à la continentalité, et d'autre part en enchâ̂nant deux microrécits, un premier, linéaire, centré sur la figure du gagnant et un second, hélicoïdal, caractérisé par la triste répétition des refondations ratées. Chacun de ces facteurs y fut certainement pour quelque chose dans l'accueil sans précédent fait à la télésérie.

Saisissant au bond la balle lancée par Benedict Anderson dans son ouvrage classique Imagined Communities ${ }^{45}$, Daniel Dayan et Elihu Katz ont développé le concept de cérémonie télévisuelle pour montrer que la télévision permet la participation collective à des moments forts et que ces cérémonies, à leur tour, contribuent à cimenter, dans leur dimension communautaire, les nations contemporaines trop vastes pour pouvoir se passer de l'« imagination nationale» de leurs membres $^{46}$. Les auteurs ont réservé leur concept à ces occasions particulières où la télévision réunit la communauté en brisant le quotidien ou la normalité. Le premier voyage du pape JeanPaul II dans sa Pologne natale, en 1979, constitue à ce titre un exemple éloquent d'une telle cérémonie ${ }^{47}$. Mais se pourrait-il qu'un concept comme celui-ci puisse aussi s'appliquer à des fictions sérielles dans les cas où ces dernières permettent à leurs fidèles d'entreprendre un armchairpilgrimage (Dayan) au cœur de leur mémoire et de leur imaginaire national et historique? 


\section{NOTES}

${ }^{1}$ L'auteur assume avec humilité chaque déficience qui pourrait miner la crédibilité de son texte et la sienne propre. Il n'en remercie pas moins avec effusion les évaluateurs de la revue Mens pour leurs nombreux commentaires qui furent, comme toujours, très utiles dans l'ensemble.

${ }^{2}$ Frédéric Demers, «La mise en scène de l'imaginaire national et historique du Québec francophone dans la télésérie Les Filles de Caleb», thèse de doctorat (histoire), Université Laval, 2004, 395 ff. La Société Radio-Canada a diffusé la télésérie entre le 18 octobre 1990 et le 28 février 1991 inclusivement, à raison d'un épisode d'une heure par semaine. Ses vingt épisodes ont attiré un peu plus de 3,66 millions de téléspectateurs en moyenne, ce qui en faisait alors le plus grand succès populaire dans l'histoire de la télévision québécoise, mais qui fut surpassé quelques années plus tard par Lá Petite Vie. Situé en Mauricie, le récit s'étalait sur les années allant de 1892 à 1917.

${ }^{3}$ Les approches de l'imaginaire sont variées. Gérard Bouchard, qui a beaucoup creusé ce sillon, a choisi d'organiser son propos sur l'opposition binaire entre culture savante et culture populaire. Yvan Lamonde a plutôt opté pour une approche géoculturelle et scruté l'influence respective de la France, de la Grande-Bretagne, des États-Unis, du Vatican et du Canada sur le Québec français. Quant à Jacques Mathieu et Jacques Lacoursière, s'inspirant du chantier ouvert en France par Pierre Nora sur les lieux de mémoire, ils ont privilégié une approche sectorielle, se penchant tour à tour sur les espaces, les grandes institutions d'encadrement, les savoir-faire, les représentations symboliques, et ainsi de suite. Des trois, l'approche sectorielle est celle qui offre le plus de souplesse. Voir Gérard Bouchard, “Une nation, deux cultures : continuités et ruptures dans la pensée québécoise traditionnelle (1840-1960) ", dans Gérard Bouchard, dir., avec la collaboration de Serge Courville, La Construction d'une culture: Le Québec et l'Amérique française, Sainte-Foy, Presses de l'Université Laval, 1993, pp. 3-47 ; id., Genèse des nations et des cultures du Nouveau Monde, Montréal, Boréal, 2000, 503 p. ; Yvan Lamonde, Allégeances et dépendances: l'bistoire d'une ambivalence identitaire, Québec, Nota Bene, 2001, 265 p. ; Jacques Mathieu et Jacques Lacoursière, Les Mémoires québécoises, Sainte-Foy, Presses de l'Université Laval, 1991, 393 p.

${ }^{4}$ Une continentalité que nous limitons à l'Amérique du Nord, car la présence de l'Amérique latine dans l'imaginaire des Québécois d'héritage canadienfrançais nous apparaît constituer un phénomène à la fois très récent et encore peu influent. 
${ }^{5}$ Cette affirmation nécessiterait de longs développements qu'il n'est pas possible d'offrir dans le cadre serré d'un texte comme celui-ci. Pour expliciter un peu, disons seulement ce qui suit. À différentes époques, la francité a tenu dans des notions comme la ruralité, la langue française, le catholicisme, la famille, l'homogénéité ethnique postulée, l'unicité du parcours historique de la nation par rapport à ses voisines, la prétendue différence essentialiste ou encore l'enracinement dans une tradition culturelle française. Il s'agit d'un usage assez proche de celui préconisé par le géographe Louis Dupont dans ses travaux. Le pôle de la canadianité réunit plutôt ces éléments particuliers du Nouveau Monde qui, d'une part, laissèrent une forte impression sur les premiers Français qui y ont séjourné et sur plusieurs générations successives d'habitants, et, d'autre part, ont constitué les facteurs les plus essentiels dans le développement, chez ces derniers, d'une identité typiquement canadienne dès le XVII e siècle. On pense à la présence de l'Amérindien, évidemment, mais aussi au nomadisme comme mode de vie, à la course dans les bois, aux espaces ouverts à l'infini, à la nordicité, à l'hiver implacable, et ainsi de suite. La continentalité, enfin, recouvre des notions qui, dans l'imaginaire franco-québécois, se sont souvent retrouvées associées à la présence anglo-saxonne : urbanisation, économie industrielle, matérialisme, capitalisme, salariat, culture de masse, volonté de participer aux grandes dynamiques continentales, etc. À cette dernière énumération, il faut bien évidemment ajouter la présence de l'anglicité au cœur du Canada français.

${ }^{6}$ C'est en vain qu'on chercherait dans notre classification une influence des travaux de Marcel Rioux. Dans un petit ouvrage des années 1970 publié en France et destiné à ce marché en priorité (Les Québécois, Paris, Seuil, 1974, 188 p.), le sociologue soutenait que l'identité québécoise combinait l'américanité, la francité et la canadianité. Pour lui, la première de ces trois notions renvoyait à tout ce qui relevait de l'adaptation au continent neuf, la seconde à la langue française et à la religion catholique, et la dernière au Canada contemporain et à sa majorité anglophone.

${ }^{7}$ Il s'agit d'une raison forte, pensons-nous, mais ce n'est certainement pas la seule. À l'origine de cette télésérie se trouve le roman du même titre signé Arlette Cousture (Montréal, Québec Amérique, 1985-1986, 2 tomes), lequel connut un colossal succès de librairie - plusieurs centaines de milliers d'exemplaires vendus - au moment de sa parution. La télésérie pouvait donc compter sur un important bassin de téléspectateurs curieux de " voir » le monde qu'ils s'étaient représenté par l'acte de lecture. À cela, il faut ajouter la qualité élevée de la production, la publicité, la critique publique emballée, le bouche à oreille, un certain effet de mode et ainsi de suite. Chose mystérieuse n'obéis- 
sant à aucune règle stricte, le succès a ses raisons que la raison parvient parfois difficilement à circonscrire avec précision.

${ }^{8}$ Sur la représentation - stéréotypée, là encore - de la ville dans Les Filles de Caleb, voir Frédéric Demers, «La ville, la campagne, l'Anglais, Les Filles de Caleb et la mémoire historique : notes sur quelques liens difficiles à démêler ", Francophonies d'Amérique, n 21 (2006), pp. 67-81.

'Sur ce plan, Henri participe d'un genre récurrent dans la fiction sérielle québécoise. Il s'inscrit dans une chaîne qui le relie en amont à Ovide Plouffe (JeanLouis Roux, La Famille Plouffe) et à Oscar Bellemare (Jean-Louis Millette, Symphorien) et en aval à Julien Constantin (Jacques L'Heureux, Virginie).

${ }^{10}$ Pour les uns, Les Filles de Caleb permettait de renouer avec l'enfance " à l'eau bénite " (Denise Bombardier) qu'ils avaient connue dans les années trente, quarante ou cinquante. Pour les autres, la télésérie ouvrait une porte sur un monde complètement révolu.

${ }^{11}$ Les Crête, ennemis d'Émilie depuis le cas de discipline impliquant leur fils Joachim (épisode 1), sont vus quittant l'école au moment même où le curé fait son discours, ce qui confirme le statut emblématique de ce dernier. Pour faire partie de la communauté, il faut nécessairement accepter la parole de l'homme d'Église. Or les Crête, parce qu'ils ne veulent pas se rallier à l'institutrice, refusent que la voix du prêtre recouvre la leur et n'ont d'autre choix que de s'exclure.

${ }^{12}$ À ce jour, la logique des choix artistiques et commerciaux qui ont été faits ne nous est pas encore parfaitement connue. Nous avons pu nous entretenir avec Arlette Cousture, auteure du roman à l'origine de la télésérie, et Fernand Dansereau, le scénariste embauché par la maison de production Cité-Amérique pour adapter le livre en scénario, mais nous n'avons pas encore eu la chance de discuter avec les producteurs, pas plus qu'avec le réalisateur de la télésérie, Jean Beaudin. Un tel entretien nous permettrait sans doute de mieux saisir la démarche de production.

${ }^{13}$ S'il fallait choisir entre la mère et l'enfant, demande par exemple le médecin à Dosithée au plus fort de l'épreuve que traverse sa femme, qui choisiriezvous ? " Le curé Grenier a toujours dit que c'est l'enfant... ", répond l'autre, hésitant. Oui, mais vous ?, insiste le médecin. Dosithée, le regard empreint de tendresse, contemple ses enfants, trop jeunes et trop nombreux pour se retrouver orphelins de mère, et tranche le dilemme : "Sauvez ma femme. » (épisode 7) Il y a la doctrine, certes, mais tout autour, il y a aussi le dur quotidien de la vie réelle. Félicitée vivra donc. 
${ }^{14}$ Une nuance : à mesure qu'elle s'agrandit, la famille fondée par Ovila et Émilie tend à devenir une sorte de facteur « objectif » de misère. Beaucoup d'enfants, ça signifie beaucoup de bouches à nourrir. Or l'argent fait souvent défaut en raison des inconstances d'Ovila en tant que pourvoyeur. La grosse famille accroit donc la pression sur le couple, rendant les situations de manque toujours plus critiques et les solutions toujours plus difficiles à identifier et à appliquer.

${ }^{15} \mathrm{La}$ famille rurale canadienne-française d'antan compose un havre de paix et un lieu de solidarité que rien ne peut troubler de manière prolongée. La télésérie martèle ce message. En ce sens, les conflits nombreux qui naissent entre Émilie et Ovila après leur mariage doivent s'interpréter non pas comme des divisions minant l'unité de la cellule familiale, mais bien comme des étincelles provoquées par l'entrechoquement de deux modèles d'organisation de la famille, l'un inspiré d'une volonté de conservation et l'autre d'une volonté de changement. Sur cette question, voir Demers, «La mise en scène... »,pp. 264-293.

${ }^{16}$ À une exception près : une élève fait un jour allusion à la liaison qu'un villageois entretiendrait avec la femme de son frère.

${ }^{17}$ Petit florilège : « Moi, ma femme [...] est pas capable de faire ça si je me rase pas. [...] Non, mais c'est fou pareil, hein ? Le bon Dieu nous agace avec ça pour que la suite du monde continue, mais on dirait qu'll agace pas nos femmes de la même manière. [...] - Je vois pas ce qu'il y a de plaisant làdedans, moi. - Tu trouves pas ça plaisant, toi ? - Ah !, c'est sale ! P'is, hein, ça sent pas toujours bon. - Peut-être que t'es plus capable de la lever ? [...] Je l'ai levée trois fois pour faire mes gars, hein ? P'is je la lèverais encore une fois sij'en voulais un quatrième. - Essayes-tu de nous faire accroire que tu l'as levée rien que trois fois ?! — En plein ça !» (épisode 15)

${ }^{18}$ Une scène se déroule en plein air dans un encan d'animaux de ferme qu'on suppose se tenir dans le village ou tout près, mais on n'y voit que des gens, pas de bâtiments (épisode 6).

${ }^{19}$ Laquelle remplit une fonction narrative toute particulière, à savoir témoigner du renversement, par Émilie, du dernier obstacle à son enracinement social dans sa communauté d'adoption, Saint-Tite. Du point de vue sémiotique, la séquence de la saynète appartient à un tout autre registre, à un tout autre récit, en fait : celui du gagnant, entamé dès les premières minutes du premier épisode, quand Émilie, adolescente, a su défier l'autorité de son père avec succès et conclu au moment de son mariage avec Ovila. Sur le microrécit 
du gagnant dans là télésérie, voir Demers, « La mise en scène... », pp. 163174.

${ }^{20}$ Sur l'indépassable conflit avec l'Anglais, voir Demers, « La ville, la campagne... », pp. 73-75.

${ }^{21}$ Entretien avec Arlette Cousture, 16 novembre 2001.

${ }^{22}$ Entretien avec Fernand Dansereau, 16 novembre 2001.

${ }^{23}$ Le Journal de Québec, 17 novembre 1990, p. $1 \mathrm{~S}$.

${ }^{24}$ Ghislaine Rheault, "Les Filles de Caleb : de la beauté avant toute chose », Le Soleil (Québec), 3 octobre 1990,p. C7.

${ }^{25}$ Daniel Rioux, "Les Filles de Caleb: un festin d'émotions!", Le Journal de Québec, 3 octobre 1990, p. 69.

${ }^{26}$ Citation tirée de Suzanne Dansereau (Presse canadienne), " Les Filles de Caleb à une heure "pour adultes" au Canada anglais ", La Presse (Montréal), 3 juin 1992, p. E3.

${ }^{27}$ Germain Monté, « Adieu Émilie et Ovila ! », Le Lundi, 9 mars 1991, article reproduit en ligne sur http:// www.iquebec.ifrance.com/marinaorsini/ article22.html, consulté le 27 août 2001.

${ }^{28}$ Ibid.

${ }^{29}$ Ibid.

${ }^{30}$ Danièle Saint-Denis, " Les Filles de Caleb », Les Personnages de Roy Dupuis, http://www.rdcharacters. com/Les\%20filles\%20de\%20Caleb.html, consulté le 27 août 2001.

${ }^{31} \mathrm{Ibid}$. Le site Internet n'est plus en fonction depuis quelques temps déjà. Le webmestre a par contre publié son contenu sous forme de livre. Voir Danièle Saint-Denis, Dans les peaux de Roy Dupuis, Montréal, Stanké, 2004, 286 p.

${ }^{32}$ Denis Massicotte, «Les Filles de Caleb, les phoques et la boucane », Le Soleil (Québec), 3 août 1991, p. A10.

${ }^{33}$ Presse canadienne, " Ovila Pronovost a maintenant sa pierre tombale à Saint-Tite », La Presse (Montréal), 16 août 1991, p. C6.

${ }^{34}$ Presse canadienne, « Le 15 août à Saint-Tite », Le Soleil (Québec), 13 juillet 1992, p. B4.

${ }^{35}$ Presse canadienne, "Les Filles de Caleb en deuil », La Presse (Montréal), 14 janvier 1993, p. C8 ; id., « La petite-fille de Caleb n'est plus », Le Soleil (Qué- 
bec), 13 février 1994, p. A2 ; «Une fille d'Émilie meurt », La Presse (Montréal), 24 février 1997, p. A3.

${ }^{36}$ Louise Cousineau, "Mort du décorateur des Filles de Caleb», La Presse (Montréal), 2 décembre 1992, p. B4.

${ }^{37}$ Mario Cloutier, «Tomber dans les pommes », La Presse (Montréal), 29 septembre 1994, p. D5.

${ }^{38}$ Citation tirée de Bruno Dostie, "Arlette Cousture tout à l'écriture d'un nouveau roman qui "raconte la vie de gens ordinaires" ", La Presse (Montréal), 27 juillet 1991,p. C7.

${ }^{39}$ Ibid.

${ }^{40}$ Citation tirée de Presse canadienne, "Ovila Pronovost a maintenant... », p. C6.

${ }^{41}$ Citation tirée de Presse canadienne, "Arlette Cousture annonce un $2^{\mathrm{e}}$ roman », La Presse (Montréal), 7 janvier 1991, p. A7. À la lumière de cette justification, on peut se demander pourquoi la ville n'a pas plutôt baptisé son édifice Arlette-Cousture.

${ }^{42}$ Royal Society of Chemistry, « Royal Society of Chemistry to Honour Sherlock Holmes with a Special Honorary Fellowship ", The Royal Society of Chemistry, http://www.rsc.org/pdf/pressoffice/2002/sherlock_holmes.pdf, consulté le 15 octobre 2002.

${ }^{43}$ "Of course Sherlock Holmes did not exist [...]. Nevertheless the value of the Holmes legend today, and in previous decades, is profound [...]. We contend that the Sherlock Holmes myth is now so deeply rooted in the national and international psyche through books, films, radio and television that he has almost transcended fictional boundaries. »Citation tirée de ibid. Dans la mesure où a réellement existé une certaine Émilie Bordeleau originaire de Saint-Stanislas, la décision de nommer la bibliothèque de cette petite municipalité en son honneur, c'est-à-dire de faire d'Émilie Bordeleau une dignitaire posthume, relève d'une logique nettement différente. La question que nous soulevions précédemment s'impose de nouveau : qui honora-t-on au juste, par ce geste? Amalgama-t-on ici, sans s'en rendre compte, le réel et la fiction ? Les édiles municipaux qui prirent la décision de baptiser une institution au nom d'Émilie Bordeleau eurent-ils su nommer une seule chose exceptionnelle que cette femme, supposément si remarquable et digne d'hommages, avait accompli de son vivant ? Si l'on ne peut pas rejeter complètement l'hypothèse que cet hommage fut le fruit gâté ? - d'une pure entreprise de récupération, le contexte nous force toute- 
fois à considérer qu'un tel cynisme n'explique pas tout. Plus probablement, le témoignage d'admiration offert à Émilie Bordeleau par la municipalité de Saint-Stanislas s'inscrivit d'abord et avant tout dans la lignée des autres manifestations de ce genre dont nous avons fait état.

${ }^{44}$ Dans la mesure où le mythe d'Émilie Bordeleau a pu naître et se déployer initialement grâce à un livre, il n'est pas banal que ce soit aussi dans un livre que la confusion entre sa personne et son personnage ait atteint son point culminant. Ce livre signé Nathalie Jean et intitulé La V raie Histoire d'Émilie Bordeleau, fille de Caleb (Outremont, Quebecor, 1995, 189 p. ; $2^{\mathrm{c}}$ éd. Boucherville, De Mortagne, 2004, 188 p.) raconte beaucoup de choses intéressantes - dans un perspective comme la nôtre, s'entend -, mais ce qu'il dit n'a que peu à voir avec la vraie histoire d'Émilie Bordeleau. Au contraire, quand vient le temps de traiter de la personnalité de son héroïne, l'auteure s'avère totalement prisonnière de l'image que la télésérie en a renvoyée. Le portrait que trace le livre de la « vraie »Émilie Bordeleau, c'est-à-dire de la personne de chair et de sang, recoupe à toutes fins utiles, pour la période qui va jusqu'à 1917, celui du personnage de la télésérie que Fernand Dansereau a créé. Ce qui a guidé l'entreprise de l'auteure fut un désir de faire correspondre la personne au personnage façonné quarante ans après sa mort. C'est un souci d'humaniser une créature de l'esprit, de lui donner consistance. Nous avons analysé assez longuement ce livre ailleurs. Voir Demers, « La mise en scène... », pp. 148155.

${ }^{45}$ Benedict Anderson, Imagined Communities : Reflections on the Origin and Spread of Nationalism, New York, Verso, 1983, 160 p.

${ }^{46}$ Daniel Dayan et Elihu Katz, La Télévision cérémonielle : anthropologie et histoire en direct, Paris, Presses universitaires de France, 1996, 259 p. (Traduction de Media Events: the Live Broadcasting of History, Cambridge, Mass., Harvard University Press, 1992, 306 p.)

${ }^{47}$ Cette observation appelle la remarque suivante. L'existence même de cette communauté, qu'on appelle "grand public » et qui dépasserait l'addition mathématique des individus postés devant leur téléviseur à un moment donné, n'est pas admise par tous. Sur ce débat, on lira en parallèle les titres suivants : Dominique Wolton, Éloge du grand public: une théorie critique de la télévision, Paris Flammarion, 1990.319 p. ; Ien Ang, Desperately Seeking the Audience, Londres, Routledge, 1990, 203 p. 